\title{
ON THE CAPACITY OF THE FADING MIMO BROADCAST CHANNEL WITHOUT CHANNEL INFORMATION AT THE TRANSMITTER AND IMPERFECT ESTIMATION AT THE RECEIVERS
}

\author{
Pablo Piantanida and Pierre Duhamel
}

\author{
Laboratoire des Signaux et Systèmes, CNRS/Supélec, \\ F-91192 Gif-sur-Yvette, France \\ Email:\{piantanida,pierre.duhamel\}@1ss.supelec.fr
}

\begin{abstract}
Consider a base station transmitting information over a downlink wireless communication channel, where the mobiles (the receivers) only dispose of a noisy estimate of the channel parameters, and these estimates are not available at the base station (the transmitter). In this paper, we examine the effects of imperfect channel estimation at the receivers and no channel knowledge at the transmitter on the capacity of the multiuser Fading MIMO Broadcast Channel. We derive the optimal Dirty-paper coding (DPC) scheme and its corresponding achievable rates with the assumption of Gaussian inputs. Our results, for uncorrelated Rayleigh fading, are particularly useful for a system designer to assess the amount of training data and the channel characteristics (e.g. SNR, fading process, number of antennas) to achieve target rates. We provide numerical results for a two-users MIMO Broadcast Channel with maximum-likehood (ML) channel estimation. These illustrate a practical trade-off between the amount of training and its impact to the multiuser interference cancellation performance. In particular, we observe the surprising result that a Broadcast Channel with a single transmitter and receiver antenna, and imperfect channel estimation at the receivers, does not need the knowledge of estimates at the transmitter to achieve large rates.
\end{abstract}

Index Terms - Broadcast channels, Maximum likelihood estimation, Channel capacity, Fading channels.

\section{INTRODUCTION}

The increasing applications of multiuser wireless communications have spawned much research about the best manner to send information to multiple users at the same time (downlink channel). In the recent years, the Gaussian Multiple-Input-Multiple-Output Broadcast Channel (MIMO-BC) has been extensively studied. Most of the literature focuses on the information-theoretic performances under the assumption that perfect channel information is available at both sides (transmitter and all receivers). In [1], the authors have established an achievable rate region, referred to as Dirty-paper coding $(D P C)$ region. They conjectured that this achievable region is the capacity. Recently in [2], this conjecture was proved by showing that the DPC region is equal to the capacity region. The most notable characteristic of the fading MIMO-BC is that under the assumption of perfect channel knowledge, as the signal-to-noise ratio (SNR) tends to infinity, the limiting ratio between the sum-rate capacity and the capacity of a single-user channel that results when the receivers are not allowed to cooperate is one. Thus, for a BC where the receivers do not cooperate, the interference cancellation implemented by DPC results in no asymptotic loss.
In most practical situations however, only a channel estimate is available at the receivers that differs from the true channel. Here we concentrate on the case where no channel information is available at the transmitter, i.e., there is no feedback from the receivers back to the transmitter conveying the channel estimates. In this scenario, it is well-known that the performances of single user channels are severely affected by the imperfect channel information at the receiver (cf. [3], [4] and [5]). Nevertheless, for multiuser channels, of particular interest is the joint effect of the imperfect knowledge at the receivers without channel information at the transmitter. In fact, the channel estimation error of one user affects the achievable rates of many other users if interference cancellation, or DPC, is used. Consequently, when the channel is not available at the transmitter and imperfectly estimated at the receivers, it is not immediately clear whether it is more efficient to send information only to one user at a time (i.e. time-division multiple-access TDMA) rather than to use multiuser interference cancellation (cf. [6]). In this matter, the study of limits of reliable information rates of the Fading MIMO-BC under these assumptions is particularly relevant. Indeed, intensive recent research has been conducted, e.g. [7] proposed an opportunistic coding scheme that employs only partial channel information. Whereas in [8], the authors show that when the transmitter only knows channel estimates, the limiting ratio between the sum-rate capacity and the capacity of a single-user channel with cooperating receivers is upper bounded by $2 / 3$. Also note that achievable rates with channel estimates available at both transmitter and all receivers have been derived in [9].

Throughout this paper we assume that the channel, which neither the transmitter nor the receivers know exactly, is estimated at each receiver by using maximum-likelihood (ML) estimation, during an independent training phase (Section 2). In this work, based on previous results [9], we derive the optimal Dirty-paper coding (DPC) scheme and its corresponding achievable rates assuming that all channel estimates are fully unknown at the transmitter (Section 3 ). We address this problem through the notion of reliable communication based on the average of the error probability over all channel estimation errors. This notion allows to change the problem to that of evaluating the achievable DPC region of a composite (more noisy) MIMO-BC. Finally, in Section 4, we use a two-users uncorrelated Rayleigh-fading MIMO-BC to illustrate average rates over all channel estimates, for different amount of training and antenna configurations.

\section{CHANNEL MODEL}

Consider a memoryless Fading MIMO-BC with $m$-users. Assume that the transmitter has $n_{T}$ antennas and each receiver has $n_{R}$ an- 
tennas, with $n_{T} \geq n_{R}$. The discrete-time channel at the time $t$ is modeled by

$$
\mathbf{y}_{k}(t)=\mathbf{H}_{k}(t) \mathbf{s}(t)+\mathbf{z}_{k}(t), \quad \mathrm{k}=1, \ldots, \mathrm{m}
$$

where $\mathbf{s}(t) \in \mathbb{C}^{n_{T} \times 1}$ is the vector of transmitter symbols and $\mathbf{y}_{k}(t) \in$ $\mathbb{C}^{n_{R} \times 1}$ is the vector of received symbols at Terminal $k$. Throughout this paper upper case boldface letters denote matrices, while lower case boldface denote vectors. Here, $\mathbf{H}_{k}(t) \in \mathbb{C}^{n_{R} \times n_{T}}$ is the complex random matrix of the Terminal $k$ whose entries are independent identically distributed (i.i.d.) zero-mean circularly symmetric complex Gaussian (ZMCSCG) random variables $C \mathcal{N}\left(0, \sigma_{h, k}^{2}\right)$. Thus, this matrix is a complex normal distributed matrix, denoted $\mathbf{H}_{k}(t) \sim C \mathcal{N}\left(0, \mathbb{I}_{n_{T}} \otimes \Sigma_{H, k}\right)$, with $\Sigma_{H, k}=\sigma_{h, k}^{2} \mathbb{I}_{n_{R}}$ the Hermitian covariance matrix of the columns of $\mathbf{H}_{k}$. The noise vector $\mathbf{z}_{k}(t) \in \mathbb{C}^{n_{R} \times 1}$ at Terminal $k$ consists in ZMCSCG random vector with covariance matrix $\Sigma_{0, k}=\sigma_{z, k}^{2} \mathbb{I}_{n_{R}}$, where $\mathbb{I}_{n_{R}}$ denotes the $n_{R} \times n_{R}$ identity matrix. We assume both $\mathbf{H}_{k}(t)$ and $\mathbf{z}_{k}(t)$ are ergodic and stationary random process, and the channel matrix $\mathbf{H}_{k}(t)$ is independent of $\mathbf{s}(t)$ and $\mathbf{z}_{k}(t)$. This leads to a stationary and discreet-time memoryless BC with marginal pdfs $\mathbf{W}_{k}\left(\mathbf{y}_{k} \mid \mathbf{s}, \mathbf{H}_{k}\right)=$ $C \mathcal{N}\left(\mathbf{H}_{k} \mathbf{s}, \Sigma_{0, k}\right), k=1, \ldots, m$. The input symbols are constrained to satisfy $\operatorname{tr}\left(\mathbb{E}_{\mathbf{s}}\left(\mathbf{s}(t) \mathbf{s}(t)^{\dagger}\right)\right) \leq \bar{P}$, where $\operatorname{tr}(\cdot)$ denotes the matrix trace and $(\cdot)^{\dagger}$ denotes Hermitian transposition.

A standard technique to allow the receivers to estimate the channel matrix consists in transmitting training sequences among the data, i.e., a set of symbols whose location and values are known to the receivers. We assume that the channel matrix is constant during the transmission of an entire codeword so that the transmitter, before sending the data $\mathbf{s}$, can teach the channel to the receivers by sending a training sequence of $N$ vectors $\mathbf{S}_{T}=\left(\mathbf{s}_{T, 1}, \ldots, \mathbf{s}_{T, N}\right)$. This sequence is affected by the channel matrix $\mathbf{H}_{k}$, allowing the receiver at the Terminal $k$ to observe separately $\mathbf{Y}_{T, k}=\mathbf{H}_{k} \mathbf{S}_{T}+\mathbf{Z}_{T, k}$, where $\mathbf{Z}_{T, k}$ is the noise matrix affecting the transmission of training symbols. The average energy of the training symbols is $P_{T}=$ $\frac{1}{N n_{T}} \operatorname{tr}\left(\mathbf{S}_{T} \mathbf{S}_{T}^{\dagger}\right)$. We focus on ML estimation of $\mathbf{H}_{k}$, for each user $k=1, \ldots, m$, from the observed signals $\mathbf{Y}_{T, k}$ and $\mathbf{S}_{T}$. This yields to $\widehat{\mathbf{H}}_{k}=\mathbf{H}_{k}+\mathcal{E}_{k}$ [9], where $\varepsilon_{k}$ denotes the estimation error matrix yielding to a white error matrix $\Sigma_{\mathcal{E}, k}=\sigma_{\mathcal{E}, k}^{2} \mathbb{I}_{n_{R}}$ and $\sigma_{\varepsilon, k}^{2}=$ $\mathrm{SNR}_{T, k}^{-1}$ with $\mathrm{SNR}_{T, k}=\frac{N P_{T}}{\sigma_{Z, k}^{2}}$, when the training sequences are orthogonal. Then, by using the fading pdf, the expression of the ML estimator and some algebra. We obtain a composite BC

$$
\widetilde{\mathbf{W}}_{k}\left(\mathbf{y}_{k} \mid \mathbf{s}, \widehat{\mathbf{H}}_{k}\right)=C \mathcal{N}\left(\delta_{k} \widehat{\mathbf{H}}_{k} \mathbf{s}, \Sigma_{0, k}+\delta_{k} \Sigma_{\mathcal{E}, k}\|\mathbf{s}\|^{2}\right),
$$

where $\delta_{k}=\frac{\operatorname{SNR}_{T, k} \sigma_{h, k}^{2}}{\operatorname{SNR}_{T, k} \sigma_{h, k}^{2}+1}$. This composite channel model is used in the next section (further details are provided in [9]).

In the following section, assuming that the channel estimates are not availables at the transmitter $\underline{\widehat{\mathbf{H}}}=\left(\widehat{\mathbf{H}}_{1}, \ldots, \widehat{\mathbf{H}}_{m}\right)$, we derive the optimal DPC scheme and its achievable rates for the Fading MIMO-BC (1). For comparison, we first review the corresponding rate region with channel estimates known at the transmitter.

\section{OPTIMAL DPC SCHEME AND ACHIEVABLE RATES}

In this paper, we use the notion of reliable communication based on the average of the error probability over all channel estimation errors $\bar{e}_{\max , k}=\mathbb{E}_{\mathbf{H}_{k} \mid \widehat{\mathbf{H}}_{k}}\left\{e_{\max , k}\left(\varphi, \phi_{k}, \widehat{\mathbf{H}}_{k} ; \mathbf{H}_{k}\right)\right\}, k=1, \ldots, m$, where $\left(\varphi, \phi_{k}\right)$ are the respective coding and decoding functions. This requires that the maximum of the averaged error probability occurs with probability less than some arbitrary small $\epsilon$ for each user, i.e., $\bar{e}_{\max , k} \leq \epsilon$. This reliability notion allows us to consider the capacity region of a composite (more noisy) BC model. A more robust notion of reliability over estimated channels is proposed in [5].

\subsection{Channel estimates known at the transmitter and all receivers}

In [9], by combining DPC scheme (as well as that proposed in [1]) and the above notion of reliable communication, the authors, assuming the channel estimates available at both transmitter and all receivers, derived the following achievable rate region.

Theorem 3.1 An achievable region $\overline{\mathscr{R}}_{T x R x}=\mathbb{E}_{\widehat{\mathbf{H}}}\left\{\widetilde{\mathscr{R}}_{T x R x}(\bar{P}, \underline{\widehat{\mathbf{H}}})\right\}$ for the Fading MIMO-BC with ML channel estimation and channel estimates known at both transmitter and all receivers, is given by

$$
\begin{gathered}
\widetilde{\mathscr{R}}_{T x R x}(\bar{P}, \underline{\widehat{\mathbf{H}}})=\operatorname{co}\left\{\bigcup \mathcal{A}\left(\pi,\left\{\mathbf{P}_{k}\right\},\left\{\widetilde{\mathbf{W}}_{k}\right\}, \underline{\widehat{\mathbf{H}}}\right)\right\}, \\
\pi,\left\{\mathbf{P}_{k}\right\}: \mathbf{P}_{k} \succeq 0 \forall k, \operatorname{tr}\left(\sum_{k} \mathbf{P}_{k}\right) \leq \bar{P}
\end{gathered}
$$

where $\mathcal{A}\left(\pi,\left\{\mathbf{P}_{k}\right\},\left\{\widetilde{\mathbf{W}}_{k}\right\}, \underline{\widehat{\mathbf{H}}}\right)=\left\{\mathbf{R} \in \mathbb{R}_{+}^{m}: R_{k} \leq \widetilde{R}_{\pi(k)}^{D P C}, k=\right.$ $1, \ldots, m\}$, and

$$
\widetilde{R}_{\pi(k)}^{D P C}=\log _{2} \frac{\left|\delta_{\pi(k)}^{2} \widehat{\mathbf{H}}_{\pi(k)}\left(\sum_{i=1}^{k} \mathbf{P}_{\pi(i)}\right) \widehat{\mathbf{H}}_{\pi(k)}^{\dagger}+\widetilde{\Sigma}_{0, \pi(k)}\right|}{\left|\delta_{\pi(k)}^{2} \widehat{\mathbf{H}}_{\pi(k)}\left(\sum_{j=1}^{k-1} \mathbf{P}_{\pi(j)}\right) \widehat{\mathbf{H}}_{\pi(k)}^{\dagger}+\widetilde{\Sigma}_{0, \pi(k)}\right|}
$$

with $\widetilde{\Sigma}_{0, \pi(k)}=\Sigma_{0, \pi(k)}+\delta_{\pi(k)} \bar{P} \Sigma_{\mathcal{E}, \pi(k)}$ and $\delta_{\pi(k)}$ defined by $\delta_{\pi(k)}=\frac{S N R_{T, \pi(k)} \sigma_{h, \pi(k)}^{2}}{S N R_{T, \pi(k)} \sigma_{h, \pi(k)}^{2}+1}$.

Let $\pi$ be a permutation on the set of indexes $\{1, \ldots, m\}$, such that $\pi$ determines the encoding order for the DPC scheme, i.e. the message of user $\pi(m)$ is encoded first while the message of user $\pi(m-$ $1)$ is encoded second and so on. The DPC region $\widetilde{\mathscr{R}}_{\mathrm{BC}}^{(\mathrm{DPC})}$ is the convex hull $\operatorname{co}\{\cdot\}$ of the union of all sets $\mathcal{A}\left(\pi,\left\{\mathbf{P}_{k}\right\},\left\{\widetilde{\mathbf{W}}_{k}\right\}, \underline{\widehat{\mathbf{H}}}\right)$ of achievable rates over all permutations $\pi$ and admissible covariance matrices $\left\{\mathbf{P}_{k}\right\}$. Here, admissibility $\mathbf{P}_{k} \succeq 0$ means that $\mathbf{P}_{k}$ is a positive semi-definite matrix and $|\cdot|$ stands for determinant.

\subsection{Channel estimates known only at the receivers}

We now focus on the optimal design of successive interference cancellation, assuming that the channel estimates are only available at the receivers, as well as in DPC scheme. A successive encoding strategy corresponds to the following approach : (i) the users are ordered (ii) each user is encoded by considering the previous users as non-causally known interference. The situation here is significantly different of that with perfect channel knowledge (cf. [1]) or when the channel estimates are also availables at the transmitter (cf. [9]). The reason is that the transmitter cannot use the channel estimates to find optimal precoding matrices for DPC scheme. In the DPC scheme, users codeword $\left\{\mathbf{s}_{k}\right\}$ with corresponding covariance matrices $\left\{\mathbf{P}_{k}\right\}$ are independent and added up to form the transmitted codeword $\mathbf{s}=\sum_{k} \mathbf{s}_{k}$. The encoder considers the interference $\widetilde{\mathbf{s}}=\sum_{i=k+1}^{m} \mathbf{s}_{i}$ due to users $i>k$ to encode the user codeword $\mathbf{s}_{k}$. The remaining codewords $\left(\mathbf{s}_{1}, \ldots, \mathbf{s}_{k-1}\right)$ are considered by the $k$ th decoder as additional noise $\sum_{i=1}^{k-1} \mathbf{s}_{i}$. Then, the $k$ th codeword $\mathbf{s}_{k}$ is obtained by letting $\mathbf{s}_{k}=\mathbf{u}_{k}-\mathbf{F}_{k} \widetilde{\mathbf{s}}$, where $\mathbf{u}_{k}$ is an auxiliary random vector chosen according to the message for the $k$ th user and $\mathbf{F}_{k} \in \mathbb{C}^{n_{R} \times n_{R}}$ is the corresponding precoding matrix. Finally, the best choice is taken among all permutations of the encoding order and assuming Gaussian inputs $\left\{\mathbf{s}_{k}\right\}$. This DPC scheme has been shown to be optimal for the MIMO-BC with perfect channel information [1]. 
Using the successive encoding strategy, we first determine an achievable rate region for the composite BC (2), which results of imperfect channel estimation at the receivers. Then, we investigate optimal precoding matrices, inspired by the optimal solution when the estimates are availables at the transmitter.

Theorem 3.2 An achievable region $\overline{\mathscr{R}}_{R x}(\underline{\mathbf{F}})=\mathbb{E}_{\widehat{\hat{\mathbf{H}}}}\left\{\widetilde{\mathscr{R}}_{R x}(\bar{P}, \underline{\widehat{\mathbf{H}}}, \underline{\mathbf{F}})\right\}$ for the Fading MIMO-BC with ML channel estimation and channel estimates not available at the transmitter, is given by

$$
\begin{aligned}
& \widetilde{\mathscr{R}}_{R x}(\bar{P}, \underline{\widehat{\mathbf{H}}}, \underline{\mathbf{F}})=\operatorname{co}\left\{\bigcup \mathcal{B}\left(\pi,\left\{\mathbf{P}_{k}\right\},\left\{\widetilde{\mathbf{W}}_{k}\right\}, \underline{\widehat{\mathbf{H}}}, \underline{\mathbf{F}}\right)\right\}, \\
& \pi,\left\{\mathbf{P}_{k}\right\}: \mathbf{P}_{k} \succeq 0 \forall k, \operatorname{tr}\left(\sum_{k} \mathbf{P}_{k}\right) \leq \bar{P} \\
& \mathcal{B}\left(\pi,\left\{\mathbf{P}_{k}\right\},\left\{\widetilde{\mathbf{W}}_{k}\right\}, \underline{\hat{\mathbf{H}}}, \underline{\mathbf{F}}\right)=\left\{\mathbf{R} \in \mathbb{R}_{+}^{m}: R_{k} \leq \widetilde{R}_{\pi(k)}^{\widetilde{D P C}}\left(F_{\pi(k)}\right),\right. \\
& k=1, \ldots, m\} \text {, and } \widetilde{R}_{\pi(k)}^{\overparen{D P C}}\left(\mathbf{F}_{\pi(k)}\right)=
\end{aligned}
$$

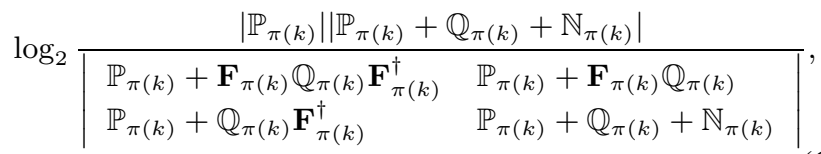

$$
\begin{aligned}
& \mathbf{P}_{\Sigma, j}^{k}=\sum_{j=i}^{k} \mathbf{P}_{j}, \\
& \mathbb{P}_{\pi(k)}=\delta_{\pi(k)}^{2} \widehat{\mathbf{H}}_{\pi(k)} \mathbf{P}_{\pi(k)} \widehat{\mathbf{H}}_{\pi(k)}^{\dagger}, \\
& \mathbb{Q}_{\pi(k)}=\delta_{\pi(k)}^{2} \widehat{\mathbf{H}}_{\pi(k)} \mathbf{P}_{\Sigma, \pi(k)+1}^{m} \widehat{\mathbf{H}}_{\pi(k)}^{\dagger}, \\
& \mathbb{N}_{\pi(k)}=\Sigma_{0, \pi(k)}+\delta_{\pi(k)} \bar{P} \Sigma_{\mathcal{E}, \pi(k)}+\delta_{\pi(k)}^{2} \widehat{\mathbf{H}}_{\pi(k)} \mathbf{P}_{\Sigma, 1}^{\pi(k)-1} \widehat{\mathbf{H}}_{\pi(k)}^{\dagger} .
\end{aligned}
$$

Actually, it remains to find the optimal precoding matrices $\underline{\mathbf{F}}=$ $\left(\mathbf{F}_{1}, \ldots, \mathbf{F}_{m}\right)$ maximizing the rates in (6). We emphasize that this maximization must be taken over all matrices not depending on the channel estimates $\underline{\widehat{\mathbf{H}}}$ (these are assumed to be unknown at the transmitter). First consider an intuitive suboptimal choice for $\mathbf{F}_{k}, k=$ $1, \ldots, m$. This choice consists in taking the average over all channel estimates of the optimal matrices with channel estimates availables at the transmitter. This amounts to the following computation

$$
\overline{\mathbf{F}}_{k}=\mathbb{E}_{\widehat{\mathbf{H}}}\left\{\mathbb{P}_{k}\left(\widehat{\mathbf{H}}_{k}\right)\left(\mathbb{P}_{k}\left(\widehat{\mathbf{H}}_{k}\right)+\mathbb{N}_{k}\left(\widehat{\mathbf{H}}_{k}\right)\right)^{-1}\right\},
$$

where $\widehat{\mathbf{H}}_{k} \sim C \mathcal{N}\left(0, \mathbb{I}_{n_{T}} \otimes \sigma_{\hat{h}, k}^{2} \mathbb{I}_{n_{R}}\right)$ with $\sigma_{\hat{h}, k}^{2}=\sigma_{\mathcal{E}, k}^{2}+\sigma_{h, k}^{2}$. By using some algebra, we can easily show the following result.

Lemma 3.3 The average in (7) is given by

$$
\begin{gathered}
\overline{\mathbf{F}}_{k}=\frac{P_{k}}{P_{\Sigma, 1}^{k}}\left[1-\rho_{k} \exp \left(\rho_{k}\right) E_{1}\left(\rho_{k}\right)\right] \mathbb{I}_{n_{R}} \\
\text { where } \rho_{k}=\frac{\sigma_{z, k}^{2}+\delta_{k} \sigma_{\varepsilon, k}^{2} \bar{P}}{\delta_{k}^{2} \sigma_{\hat{h}, k}^{2} \mathbf{P}_{\Sigma, 1}^{k}} \text { and } E_{1}(z)=\int_{z}^{\infty} t^{-1} \exp (-t) d t d e- \\
\text { notes the exponential integral function. }
\end{gathered}
$$

The other (obviously optimal, but solvable numerically only) possibility is to find directly the optimal matrix $\mathbf{F}_{k}^{*}$ maximizing (6). Observe that

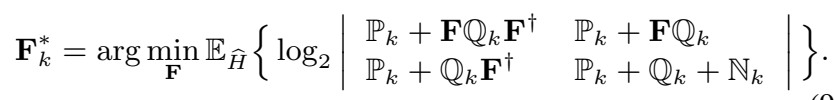

Therefore, by using some algebra and after factorizing the matrix $\widehat{\mathbf{H}}_{k}$. The solution of (9) is shown to be given by $\mathbf{F}_{k}^{*}=\alpha_{k}^{*} \mathbb{I}_{n_{R}}$,

$$
\begin{aligned}
\alpha_{k}^{*} & =\arg \min _{0 \leq \alpha \leq 1}\left\{\lambda ( \alpha ) \left[\exp \left(\frac{\beta_{-, k}(\alpha)}{4 \alpha}\right) E_{1}\left(\frac{\beta_{-, k}(\alpha)}{4 \alpha}\right)\right.\right. \\
& \left.\left.-\exp \left(\frac{\beta_{+, k}(\alpha)}{4 \alpha}\right) E_{1}\left(\frac{\beta_{+, k}(\alpha)}{4 \alpha}\right)\right]\right\},
\end{aligned}
$$

and $\lambda(\alpha)=\frac{a_{0, k} a_{1, k}^{-1}}{a_{3, k} \sqrt{b_{k}^{2}-4 \alpha}}, \beta_{ \pm, k}(\alpha)=b_{k} \pm \sqrt{b_{k}^{2}-4 \alpha}, b_{k}=$ $\frac{a_{0, k}}{a_{1, k} a_{3, k}}\left(\frac{2 a_{1, k} a_{2, k}}{a_{0, k}}-1\right), a_{0, k}=\left(\delta_{k}^{2} \mathbf{P}_{k}+\delta_{k}^{2} \mathbf{P}_{\Sigma, k+1}^{m} \alpha\right)^{2}, a_{1, k}=$ $\delta_{k}^{2} \mathbf{P}_{k}+\delta_{k}^{2} \mathbf{P}_{\Sigma, k+1}^{m} \alpha^{2}, a_{2, k}=\delta_{k}^{2} \bar{P}$ and $a_{3, k}=\sigma_{z, k}^{2}+\delta_{k} \sigma_{\mathcal{E}, k}^{2} \bar{P}$. Unfortunately, (10) does not lead to an explicit solution for $\alpha_{k}^{*}$. However, this maximization can be numerically solved for each $k=$ $1, \ldots, m$, to compute $(6)$ and then $\overline{\mathscr{R}}_{\mathrm{Rx}}\left(\underline{\mathbf{F}}^{*}\right)$. Both solutions were tested, and we observed that the achievable rates with $\underline{\overline{\mathbf{F}}}$ are very close to those provided by the optimal solution $\mathbf{F}^{*}$. Curves are omitted due to lack of space. As a result, we have chosen in the simulations below to use the mean parameter to design the "close to optimal" DPC scheme.

Note that, under channel estimation errors, the channel noise of the resulting composite $\mathrm{BC}$ (2) is correlated to the channel input. Thus, its probability distribution is actually depending on the probability distribution of the input $\mathbf{s}$. As a consequence, non Gaussian input distributions are expected to attain the boundary points of the capacity region. Therefore, our assumption of Gaussian inputs only leads to an achievable rate region, which is not the capacity region.

\section{SIMULATION RESULTS AND DISCUSSIONS}

In this section, we illustrate our results via a realistic downlink wireless communication scenario involving a two-users $(m=2)$ Fading MIMO Broadcast Channel (1). Consider first that the base station and the mobiles have a single antenna $\left(n_{T}=n_{R}=1\right)$. We show the average (over all channel estimates) of achievable rates with ML channel estimation (5) and channel estimates unknown at the transmitter, for different amount of training $N$. For comparison, we also show similar plots with channel estimates known at the transmitter, the time-division rate region where the transmitter sends information to only a single user at a time and the ergodic capacity under perfect channel information. We assume that the transmitter must satisfy the short-term power constraint $\bar{P}$, for every fading state. Then, we investigate the evolution of achievable rates by increasing the number of transmitter and receiver antennas. To this end, we consider a transmitter with four antennas $\left(n_{T}=4\right)$ and receivers with two antennas $\left(n_{R}=2\right)$.

Fig. 1 shows the average of the achievable rate region (in bits per channel use) with estimated channel information at both transmitter and all receivers (Theorem (3.1)) and with channel estimates only availables at the receivers (Theorem (3.2)), for different amount of training $N=\{5,20\}$. We suppose very different signal-to-noise ratios $\mathrm{SNR}_{1}=0 \mathrm{~dB}$ and $\mathrm{SNR}_{2}=10 \mathrm{~dB}$, and equal fading distributions $\sigma_{h_{1}}^{2}=\sigma_{h_{2}}^{2}=1$. Here, the training assumes same channel SNR, i.e., the average energy of the training symbols is $P_{T}=\bar{P}$. Observe that the achievable rates with channel estimation are still quite large irrespective of the small training sequence length $N=5$ (dashed and danshed-dot lines), i.e. 0.2 bits less comparing to the capacity with perfect channel information (solid line). Suppose now that user-2 needs to send information at a rate $R_{2}=1.5$ bits. We want to determine, how large performance gains can be achieved for user- 1 , when the channel estimates are not availables at the transmitter. We investigate this by observing the gain for the first user when the second user is transmitting at 1.5 bits. Note that this gain is -0.1 bits (with $N=20$ ) and -0.22 bits (with $N=5$ ) less compared to the case of perfect channel information. On the other hand, only 0.04 bits more are expected when the transmitter knows the channel estimates. This rate gain is slightly smaller, and consequently we can conclude that the knowledge of the channel estimates at the transmitter is not really necessary with the proposed DPC scheme. 
Fig. 2 shows similar plots with $n_{T}=4$ and $n_{R}=2$. In this multiple antenna scenario, without channel information at the transmitter, there can be no adaptive spatial power allocation. However, at equal power, it is seen that a small increase in the number of transmitter antennas can cause significant improvement, comparing with the single antenna case. Note that our short-term power constraint is averaged over all transmitter antennas, so that this power constraint is independent of the number of transmitter antennas. Consider now that user- 2 needs to send information at a rate $R_{2}=5$ bits. We observe that, with channel estimates availables at the transmitter, significant gains can be achieved compared to the case where the estimates are unknown at the transmitter (approximately 2 bits). Whereas, a multiple antenna Broadcast Channel achieves rates close to those of the time-division multiple access (dot line). The gain, by using DPC instead of TDMA, is reduced to only 0.12 bits with $N=20$, while not signicative gain is observed for $N=5$. Note that this gain is equal to that obtained with a single antenna. Therefore, for MIMO-BC, taking a real benefit from a large number of transmit antennas would require an instantaneous knowledge of channel estimates at the transmitter. If it is not the case, TDMA provides similar performances to MIMO Broadcast channels.

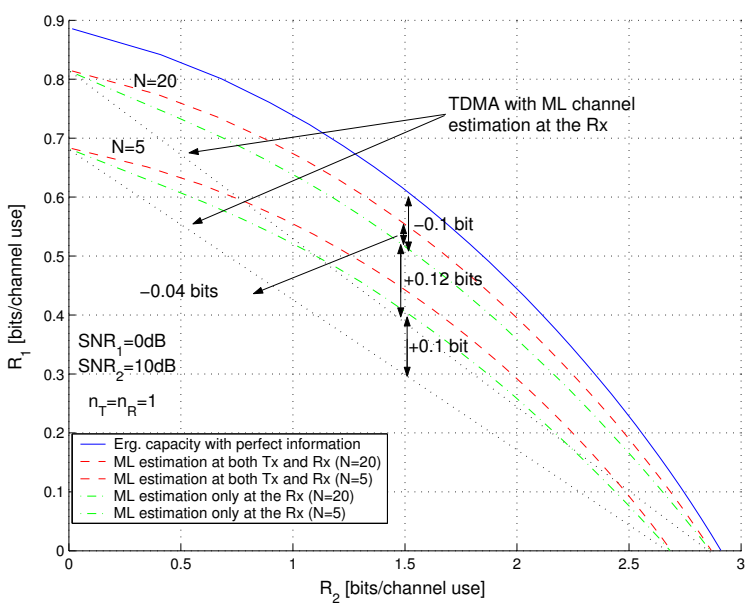

Fig. 1. Achievable rates with a single antenna $\left(n_{T}=n_{R}=1\right)$ and channel estimates known at the transmitter (dashed lines) vs the SNR, for training sequence lengths $N=\{5,20\}$. Dashed-dot lines suppose channel estimates unknown at the transmitter. Solid line shows the capacity with perfect channel knowledge.

\section{CONCLUSION}

In this paper we studied the problem of reliable communication over uncorrelated Fading MIMO Broadcast Channels. We assumed that no channel information is available at the transmitter and the receivers only have access to a noisy estimate of the channel. In this scenario, we characterized an achievable rate region with ML channel estimation. We derive the optimal Dirty-paper coding (DPC) scheme under the assumption of Gaussian inputs. Our results, for downlink communications, are useful to assess the amount of training data to achieve target rates. These show that a BC, with a single transmitter and receiver antenna and no channel information at the transmitter, can still achieve significant gains using the proposed DPC scheme. Further numerical results show that, under the assumption of imperfect channel information at the receiver, the benefit of channel estimates known at the transmitter does not lead to large rate increases. However, we also showed that, for multiple antenna $\mathrm{BCs}$, in order to achieve large gain rates using multiuser interference cancellation instead of TDMA, the transmitter requires the knowledge of all channel estimates, i.e., some feedback channel (perhaps rate-limited) must go from the receivers to the transmitter, conveying these channel estimates.

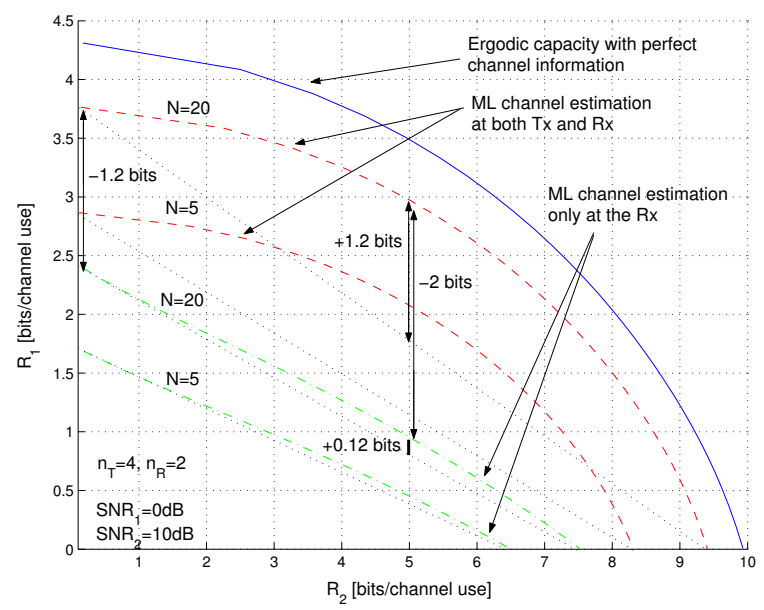

Fig. 2. Similar plots of achievable rates, with four transmitter antennas $\left(n_{T}=4\right)$ and two receiver antennas $\left(n_{R}=2\right)$.

\section{REFERENCES}

[1] G. Caire and S. Shamai, "On the achievable throughput of a multi-antenna gaussian broadcast channel," IEEE Trans. Information Theory, vol. IT-49, pp. 1691-1706, july 2003.

[2] H. Weingarten, Yossef Steinberg, and S. Shamai, "The capacity region of the gaussian MIMO broadcast channel," in To appear in IEEE Trans. on Information Theory, presented in ISIT 2004.

[3] M. Médard, "The effect upon channel capacity in wireless communication of perfect and imperfect knownledge of the channel," IEEE Trans. Information Theory, vol. IT-46, no. 3, pp. 933-946, May 2000.

[4] Taesang Yoo and Andrea Goldsmith, "Capacity of fading MIMO channels with channel estimation error," in Proceedings of International Conf. on Comunications (ICC), June 2004.

[5] P. Piantanida, G. Matz, and P. Duhamel, "Outage behavior of discrete memoryless channels under channel estimation errors," in Proc. of International Symposium on Information Theory and its Applications, ISITA 2006, October 2006.

[6] N. Jindal and A. Goldsmith, "Dirty paper coding versus TDMA for MIMO broadcast channels," IEEE Trans. Information Theory, vol. 5, pp. 1783-1794, May 2005.

[7] M. Sharif and B. Hassibi, "On the capacity of MIMO broadcast channel with partial side information," IEEE Trans. Information Theory, vol. 51, no. 2, pp. 506- 522, Feb. 2005.

[8] A. Lapidoth, S. Shamai, and M. Wigger, "On the capacity of a MIMO Fading Broadcast Channel with imperfect transmitter side-information," in Proceedings of Allerton Conf. on Commun., Control, and Comput., Sep. 2005.

[9] P. Piantanida and P. Duhamel, "Achievable rates for the Fading MIMO Broadcast Channel with imperfect channel estimation," in Proc. of the Forty-Fourth Annual Allerton Conference on Communication, Control, and Computing, Sep. 27-29 2006. 